\title{
Investment Casting Queuing Procedure
}

\author{
R. Vanalakshmi, S. Maragathasundari, S. Kishore Eswar
}

\begin{abstract}
Investment casting is a modern procedure dependent on lost-wax throwing, which is one of the most seasoned known metal-farming methods. Investment casting gets its name from the example being contributed (encompassed) with a headstrong material. The process carried out in Investment casting is well investigated by means of Queuing concept. By the usage of supplementary variable technique, the Queuing issue in investment casting is solved and its corresponding probability generating queue size and all its execution measures are determined. Numerical and graphical study throws eminent recognition for the queuing model under study.

Keywords: Delay, Stage interruption, balking, Reneging, Investment casting.
\end{abstract}

\section{INTRODUCTION}

$\mathrm{I}$ nvestment casting has been used in various structures all through the previous years. In its most punctual structures, beeswax was utilized to shape designs vital for the casting procedure. Now days for making designs, developed waxes, unmanageable material and other combination of materials are used. Investment casting is esteemed for its capacity to create segments with exactness, repeatability, flexibility and honesty in an assortment of metals and elite amalgams. "Reference [1] connected the computerized assembling casting concentrating on the procedure chain as opposed to plastic example utilized in investment casting giving a role as expandable material". "Reference [3] proposed an engineering and framework modules for a major information investigation stage to actualize brilliant production lines and medium measured enterprises". "Reference [8] built up a half and half investment casting process for modern applications". "Reference [9] created a wax mix which could offer better surface completion, least shrinkage and moderate hardness". "Reference [10] built up a procedure fusing neutron radiography". "Reference [11] examined the most recent creative plans to improve and qualities of the traditional speculation throwing process. "Reference [12] displayed an investigation of plastic example utilized in

Revised Manuscript Received on December 5, 2019

* Correspondence Author

R. Vanalakshmi , Department of Mathematics, Kalasalingam Academy of Research and Education, Krishnankovil-626126, Tamilnadu. Email: vanalakshmi31@gmail.com

S. Maragathasundari*, Department of Mathematics, Kalasalingam Academy of Research and Education, Krishnankovil-626126, Tamilnadu. Email: maragatham01@gmail.com

S. Kishore Eswar, Department of Mechanical Engineering, Kalasalingam Academy of Research and Education, Krishnankovil-626126, Tamilnadu.Email: kishoreeswar333@gmail.com Paradigm to one-off vacuums helped quick investment on engineering issues". "Reference [2] considered the

investment casting giving a role as an expandable material. "Reference [13] outlined the earthenware advancements at present being actualized to investigate the potential techniques for the executes of shell execution. "Reference [4] studied a $M[x] / G / 1$ Queue with Bernoulli schedule, General vacation and general repair times. "Reference [5] worked on Mobile adhoc networks problem through queuing approach. "Reference [6] investigated a single server queue with optional phase type server vacations based on exhaustive deterministic service and a single vacation policy. "Reference [7] analyzed a M[x]/G/1 queue with Bernoulli vacation schedule under restricted admissibility policy.

Numerous materials are reasonable for Investment casting precedents are hardened steel amalgams, metal, aluminum, carbon steel and glass. The material is filled a depression in a recalcitrant material that is a careful copy of the ideal part. Because of the hardness of headstrong materials utilized, speculation throwing can deliver items with remarkable surface characteristics, which can decrease the requirement for optional machine forms.

\section{A. Master pattern}

A craftsman or shape producer makes a unique example from wax, plastic, clay, wood or another material. As of late the generation of examples utilizing three dimensional printing has turned out to be prominent utilizing either standard PLA fiber or specially designed 'throwing wax' fiber.

\section{B. Mould preparation}

A shape, defined as the ace bite the dust, is made to fit the ace example. On the off chance that the ace example was produced using steel, the ace pass on can be thrown straightforwardly from the example utilizing metal with a lower softening point. Elastic molds can likewise be thrown straightforwardly from the ace example. On the other hand, an ace pass on can be machined autonomously-without making an ace example.

\section{Produce wax designs}

Wax precedents can be given in two distinct behaviors. In the first strategy, the wax is filled the structure and washed around until a despite covering, generally around $3 \mathrm{~mm}$ broad, bounds the internal surface of the shape. Alternative scheme incorporates filling the entire shape with fluid wax and allowing it to cool as a solid thing.

\section{Accumulate wax designs}

Multiple wax models can be made and gathered into one immense guide to be tossed in one pack pour. In this situation, structures are affixed to a wax goad to make a model gathering, or tree. 
To associate plans, a warming instrument is used Upwards of a couple of hundred models can be gathered into a tree.

The shape (wax design) is been set up for the approaching liquid wax. The liquid wax is been set up with the assistance of heater. As a rule in all assembling businesses the pouring holder of the liquid wax will be in bigger volume. In this way the cavity will be in littler volume. The temperature of the wax in the holder must be kept up.

\section{E. Queuing Issue}

The above process of study meeting various concepts is converted in terms of queuing problem. The problem is investigated and solved by supplementary variable technique. This method deals with the comparing Queuing execution proportions of the procedure did in Investment Casting strategy. Queuing issues in casting explained in terms of Queue parameters are defined below.

- Balking: In spite of the fact that the pouring pit is littler volume, all the wax from the compartment won't be poured completely from holder. Some of liquid wax material will be staying in the compartment. There emerges the recoiling factor. Where a portion of the wax material is stayed in the holder (not all the wax materials will be filled the pit).

- Stage 1: Form planning for making a wax design. Essentially these wax designs are made utilizing infusion forming process. This procedure requires infusion forming machine, crude material and the shape. The plastic is liquefied in the embellishment machine and after that infused into the shape, where it cools and cements into the last way. Essentially the form is set up by considering the ace example materials. A few organizations attempt the ace example utilizing wax and plastics at the same time. In this manner the arrangement of the showstopper shape would take longer time. While setting up the shape once ought to consider the stipends, for example, Shrinkage or Contraction recompenses, Draft or Taper remittances, Machining or Finishing recompense and Shake remittance.

- Service interference: In view of the work piece, the gated example material will fluctuate. Material we use for gated design material will choose the precision of the completed item. Numerous enormous scale businesses will make numerous items. They fabricate items with higher precision rate and furthermore they produce in lower exactness state. They make the two sorts of items all the while. In this manner, the material that we are going to use in the entryway example will fluctuate. For the most part for low precision they use wax as a material and for higher exactness they use plastics as a material. Since enterprises fabricate both sort of items, arrange one procedure will be ceased and in the wake of setting the material, further procedure will happen.

- Delay process: Since, we are changing the gated example material dependent on their exactnesses, the procedure will devour additional time as we are simply going to guarantee the components of the pit, cavity plan, cementing element and number of little material example that will be suited in the fundamental branch.

- Repair process: Here in this procedure we are going to fix the more seasoned structure of the material. The structure of specific material won't exist in another material. Henceforth, we have to fix the more established structure that is been utilized. The elements like Fluidity, Shrinkage, Resistance to hot tears and cast ability rating assumes a noteworthy job in fixing process. Most significant factor is the Allowance given to material. Stipends like Shrinkage, Taper, Shake, and so on;

- Stage 2: Arrangement of wax design into a gated example group: Once in a while, the quantity of wax design in the gated example will be decreased. The decrease of wax design (or) the quantity of course of action of the wax design is chosen distinctly by pouring liquid metal. Now and again, the clients would incline toward for a high solidify material. Around then, the organization will change the pouring liquid metal. In light of pouring liquid metal and thinking about the recompenses, they lessen or organize of the wax design. Generally in venture throwing process, this assumes a noteworthy job since this stage is going to go about as pit in further procedures. In the event that the quantity of wax design is high, the cementing of the liquid metal happens ill-advised or halfway all through the pit. Insignificant number of wax example will likewise influence the hardening procedure in light of the fact that the time factor is being expanded. By thinking about every one of these elements, the plan of wax design is resolved.

- Reneging: Some times, the quality of materials may change part to segment. Variety in quality of segment will happen dependent on pouring liquid metal on the pit where the hardening of material contrasts. Cementing assumes a noteworthy job on the material organization. Cementing can be dictated by the course of action of the wax design. The number wax example will be masterminded in an arrangement in spite of the fact that the wax design is further proselyte into pit or form. The number wax design is being masterminded dependent on use of the pouring metal. Hence remaining wax design are been left for the future procedure. This procedure is been named as reneging process.

\section{NOTATIONS}

(1) () - Steady state probability that there are $\mathrm{n}$ customers in the Queue excluding the customers in first stage of service and the elapsed service time of this is $\mathrm{x}$.

(2) () - Steady state probability that there are n customers in the Queue excluding the customers in second stage of service and the elapsed service time of this is $\mathrm{x}$.

()- The server is inactive due to a system break down and is waiting for repairs to start with elapsed delay time $\mathrm{x}$. This also represents the steady state.

()- The server is under repair irrespective of the value of $\mathrm{x}$. This denotes the steady state probability in which there are $n$ customers in the queue.

\section{MATHEMATICAL ASSUMPTIONS OF THE MODEL}

Let ${ }^{(1)}()$ be the conditional probability of stage 1 service with distribution function ${ }^{*}(\mathrm{x})$ and density function () and it is given by

${ }^{(1)}()=\frac{O}{1-{ }^{*}(\mathrm{x})}$ and $(\mathrm{x})={ }^{(1)}()\left[-\int_{0}{ }^{(1)} \mathrm{O}\right]$

Likewise, for the various parameters, we have 
${ }^{(2)} \mathrm{O}=\frac{h()}{1-^{*}(\mathrm{x})}$ and $h(\mathrm{x})={ }^{(2)}()\left[-\int_{0}{ }^{(2)} \mathrm{O}\right]$

$\mathrm{O}=\frac{\mathrm{O}}{1-()}$ and e $\left.\mathrm{x}\right)=\left(\mathrm{O}\left[-\int_{0} \mathrm{O}\right]\right.$

()$=\frac{O}{1-{ }_{3}^{*}(0}$ and $(x)=0\left[-\int_{0}()\right]$

Customers arrive in batches follows a Poisson distribution. During the first stage of service, break down occurs. Arrival of break down follows a Poisson stream. The concept of Balking occurs during the first stage of service with the parameter ${ }_{1}$. Due to impatience the customers leave the system during the second stage of service after joining the queue. This concept is defined to be Reneging. Reneging is assumed to follow exponential distribution with parameter.

\section{GOVERNING EQUATIONS OF THE MODEL}

According to the assumptions mentioned in the previous section, the following set of equations represents the queuing system we study in this paper. By the usage of quasi birth and death process, the equations are framed.

${ }^{(1)} \mathrm{O}+\left(+{ }^{(1)} \mathrm{O}+\right)^{(1)} \mathrm{O}={ }_{1} \sum_{=1}^{\infty}{ }^{(1)}()+\left(1-{ }_{1}\right)^{(1)}()$

(1)

${ }_{0}^{(1)} \mathrm{O}+\left(+{ }^{(1)}()+\right)_{0}^{(1)} \mathrm{O}=\left(1-{ }_{1}\right)_{0}^{(1)} \mathrm{O}$.

(2)

${ }^{(2)} \mathrm{O}+\left(+{ }^{(2)} \mathrm{O}+\right)^{(2)} \mathrm{O}=\sum_{=1}^{\infty}{ }^{(2)} \mathrm{O}+{ }_{+1}^{(2)} \mathrm{O}$

(3)

$\mathrm{O}+(+\mathrm{O}) \mathrm{O}=\sum_{=1-}()$

${ }_{0} \mathrm{O}+(+())_{0} \mathrm{O}=0$.

$\mathrm{O}+(+())\left(0=\sum_{=1-}()\right.$.

${ }_{0}()+(+())_{0}()=0$.

$=\int_{0}^{\infty}{ }_{0}() O+\int_{0}^{\infty}{ }_{0}^{(2)} \mathrm{O}^{(2)}()$.

Boundary condition

${ }^{(1)}(0)=\int_{0}^{\infty}{ }^{(2)}()^{(2)}()+\int_{0}^{\infty}+1()()+{ }_{+1}$.

(10)

${ }^{(2)}(0)=\int_{0}^{\infty}(1)()^{(1)}()$.

(0) $=\int_{0}^{\infty}{ }_{-1}^{(1)}()={ }_{-1}$.

$(0)=\int_{0}^{\infty}()($.

\section{DISTRIBUTION OF THE LINE LENGTH}

Solving of (1) to (8)

We multiply (1) by and sum over from 1 to $\infty$, and add it to (2) resulting in the following equation.

${ }^{(1)}()+,\left({ }_{1}(1-())+{ }^{(1)}()+\right)^{(1)}()=$,0 .

(14)

${ }^{(2)}()+,\left(-0+-+{ }^{(2)}(0){ }^{(2)}()=\right.$,0 .

(15)
$()+,(-0)+(0)()=$,0 .
$()+,(-0+0)()=$,0 .

Multiply (10) by ${ }^{+1}$ and summing over from 0 to $\infty$, we obtain

${ }^{(1)}(0)=,\int_{0}^{\infty}(2)(,)^{(2)}()+\int_{0}^{\infty}() 0+0-,\left[\int_{0}^{\infty}{ }_{0}^{(2)}(,)^{(2)}()+\right.$ $0 \infty 0$,

Using (9) in (18), we get
${ }_{0}^{(2)} \mathrm{O}+\left(+{ }^{(2)} \mathrm{O}+\right)_{0}^{(2)} \mathrm{O}={ }_{1}^{(2)} \mathrm{O}$.

${ }^{(1)}(0)=,\int_{0}^{\infty}(2)(,)^{(2)}()+\int_{0}^{\infty}()()+,(0-1)$.

(19)

Applying the same for (11), (12) and (13), we get

${ }^{(2)}(0)=,\int_{0}^{\infty}(1)(,)^{(1)}()$

$(0)=,\int_{0}^{\infty}(1)()=,{ }^{(1)}()$.

$(0)=,\int_{0}^{\infty}()()$, .

Integrating (14) from 0 to, yields

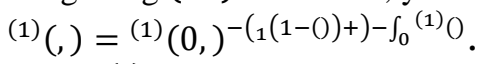

Where ${ }^{(1)}(0$,$) is given by (19)$

Let $={ }_{1}-{ }_{1}()+$

Integrating (23) by parts with respect to yields,

${ }^{(1)} \mathrm{O}={ }^{(1)}(0),\left(\frac{\left.1-{ }^{*}\right)}{}\right)$.

Where ${ }^{*}()=\int_{0}^{\infty}-\left({ }_{1}(1-0)+\right)(O)$ is the Laplace Stieltje's transform of the service time ().

Multiply both sides of (23) by ${ }^{(1)}()$ and integrating over, we get

$\int_{0}^{\infty}(1)(,)^{(1)}()={ }^{(1)}(0,)^{*}()$.

Using equation (25) in (20), we get

${ }^{(2)}(0)=,{ }^{(1)}(0,)^{*}()$

Likewise integrating (15) and applying the same process we get

${ }^{(2)}()={ }^{(2)}(0),\left(\frac{1-{ }^{*}}{}\right)$.

(26)

$\int_{0}^{\infty}(2)(,)^{(2)}()={ }^{(1)}(0,)^{*}()^{*}()$.

Where $=-()+-$

For Delay process, we have

$O={ }^{(1)}(0),\left(\frac{1-^{*} O}{)}\right)\left(\frac{1-O}{)}\right.$.

$\int_{0}^{\infty}() 0=,{ }^{(1)}(0),\left(\frac{1-^{*}()}{}\right)()$.

For repair process we have,

()$={ }^{(1)}(0),\left(\frac{1-^{*} O}{)}\right)\left(\frac{1-0}{)}\right)$.

$\int_{0}^{\infty}() O=,{ }^{(1)}(0),\left(\frac{1-^{*}()}{}\right)()()(31)$

Now substituting (27) and (31) in (19), we get

${ }^{(1)}(0)=,{ }^{(1)}(0,)^{*}()^{*}()+{ }^{(1)}(0),\left(\frac{1-{ }^{*}()}{}\right)()(0+(0-1)$

(1) $(0)=,\frac{(0-1)}{-{ }^{*}()^{*}()-\left(\frac{\left.1-{ }^{*}\right)}{}\right) O O}$.

(32) Substituting

for ${ }^{(1)}(0$,$) in (24), (26), (28) and (30), we get,$

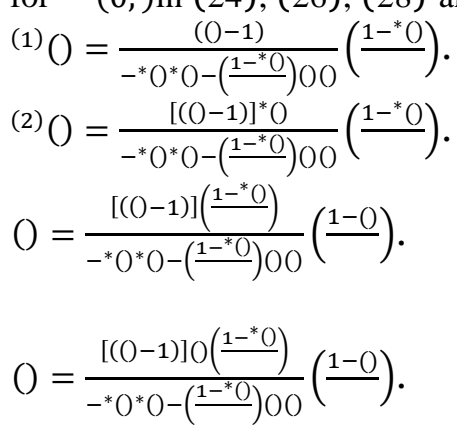

\section{PROBABILITY GENERATING FUNCTION OF THE QUEUE SIZE}

Let ${ }^{*}()$ be the chance making function of the queue (i.e.) ${ }^{*}()={ }^{(1)}()+{ }^{(2)}()+()+()$.

Thus adding (33) - (36), we get 
$[(0-1)]\left[\left(\frac{1-{ }^{*}(}{)}\right)++^{*} O\left(\frac{\left.1-{ }^{*}\right)}{)}\right)\right]$
${ }^{*}()=\frac{-\left(1-{ }^{*}()\right)\left[\left(\frac{1-O}{)}\right)+O\left(\frac{1-O}{)}\right)\right]}{\left.-^{*}\right)^{*}\left(O-\left(\frac{1-*}{)}\right) O O\right.}$.

The normalization condition ${ }^{*}(1)+=1$, is used to determine

L. H rule is applied on (37) to obtain

$\lim _{\rightarrow 1} * O=\frac{{ }^{(1)}}{{ }^{(1)}}$

(38)

Next, we obtain idle time

$$
\begin{aligned}
& =\frac{{ }^{\prime}(1)}{(1)+{ }^{\prime}(1)} \\
& 1+{ }^{*}()-{ }^{*}\left(\mathrm{O}(-+)()-\left(\frac{1-{ }^{*}()}{}\right)-*^{*}(\right. \\
& =\frac{-\left(\frac{1-^{*} 0}{)}\right)(0+0)}{1+^{*}\left(0-^{*}\right)(-+) 0-\left(\frac{\left.1-^{*}\right)}{}\right)-*^{\prime}()-} \text {. } \\
& \left(\frac{1-*}{}\right)(0+0) \\
& +\mathrm{O}\left(\frac{1-^{*} 0}{}\right)-\left(1-{ }^{*} \mathrm{O}\right)(\mathrm{O}+\mathrm{O})
\end{aligned}
$$

. From (39) the utilization factor can be found out.

\section{SYSTEM QUEUE EXECUTION PROCEDURES}

Using L.H rule twice we obtain $=\lim _{\rightarrow 1} \frac{{ }^{\prime \prime}\left(\mathrm{O}-{ }^{\prime}()^{\prime \prime}()\right.}{2\left({ }^{\prime}()^{2}\right.}$. (40)

Finding the derivative at $=1$, we have

$(1)=0\left(\frac{1-^{*} 0}{}\right)-\left(1-{ }^{*}(0)(0+0)\right.$.

(41)

$$
{ }^{\prime \prime}(1)=\left[*^{\prime}\left(0+{ }^{*}(0)\right](2)-\left[\left(1-{ }^{*}(0)(0+0)+{ }_{1}{ }^{\prime}()+\right.\right.\right.
$$$$
1-*++*^{\prime}++1-* 2+(2)+2() \text {. (42) }
$$$$
{ }^{\prime}(1)=1+{ }^{*}()-{ }^{*}()(-+)()-\left(\frac{\left.1-{ }^{*}\right)}{}\right)-{ }^{*}()-
$$$$
\left(\frac{1-^{*} 0}{)}\right)(0+0) \text {. }
$$

"(1) $=-$ * $^{\prime \prime}()^{2}+{ }^{*}()()(-+)+{ }^{*}() O(-+)-$ ${ }^{*}()\left({ }^{2}\right)(-+)^{2}+2^{*} O()-\left[\frac{*^{\prime} O}{+}+\left(\frac{1-^{*} O}{)}\right)(0+0)\right]-$ $\left[*^{\prime}\left(0-*^{\prime \prime}()-*^{\prime}(0(0)+0)\right]-\left[\left(\frac{1-{ }^{*} 0}{)}\right)(0+0)-\right.\right.$ $*^{\prime}++1-* 22+2+2()$.

\section{(44)}

Substituting (41) - (44) in (40) is attained.

Using little's formula we further get,

$=+,=,=$.
VIII. NUMERICAL JUSTIFICATION OF THE MODEL

$1=1,{ }^{*}()=\frac{(1)}{(1)_{+}},{ }^{\prime}()=\frac{-(1)}{\left((1)_{+}\right)^{2}},()=\frac{1}{},\left({ }^{2}\right)=\frac{2}{2},()=$

$\frac{1}{-},\left({ }^{2}\right)=\frac{2}{2},()=\frac{1}{(2)},\left({ }^{2}\right)=\frac{2}{\left({ }^{(2)}\right)^{2}},{ }^{\prime \prime}()=\frac{2^{(1)}}{\left({ }^{(1)}+\right)^{3}},=3,(1)=$

$4,{ }^{(2)}=5,=2,=2.5,=3.5$

Table-I: Effect of Change Of $\alpha=1.5,1.6,1.7,1.8,1.9$

\begin{tabular}{|c|c|c|c|c|l|}
\hline $\mathrm{Q}$ & $\rho$ & $\mathrm{L}_{\mathrm{q}}$ & $\mathrm{L}$ & $\mathrm{W}_{\mathrm{q}}$ & \multicolumn{1}{|c|}{$\mathrm{W}$} \\
\hline 0.2999 & 0.7001 & 26.0344 & 26.7345 & 8.6781 & 8.9115 \\
\hline 0.2899 & 0.7101 & 41.9803 & 42.6904 & 13.9934 & 14.2301 \\
\hline 0.2778 & 0.7222 & 55.7088 & 56.4310 & 18.5696 & 18.8103 \\
\hline 0.2577 & 0.7423 & 94.4130 & 95.1552 & 31.4710 & 31.7184 \\
\hline 0.2246 & 0.7754 & 221.9618 & 222.7372 & 73.9873 & 74.2457 \\
\hline
\end{tabular}

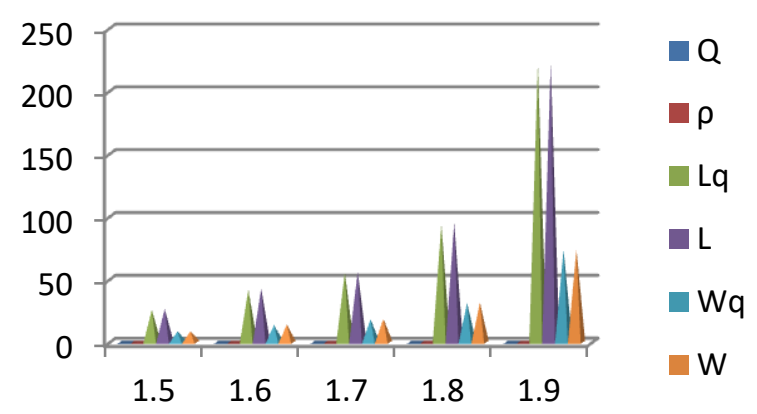

Fig 1: Effect of change of $\alpha$

Table-II: Effect of Change Of $\gamma=3.5,4,4.5,5,5.5$

\begin{tabular}{|c|l|l|l|l|l|}
\hline $\mathrm{Q}$ & \multicolumn{1}{|c|}{$\mathrm{P}$} & \multicolumn{1}{c|}{$\mathrm{L}_{\mathrm{q}}$} & \multicolumn{1}{c|}{$\mathrm{L}$} & $\mathrm{W}_{\mathrm{q}}$ & $\mathrm{W}$ \\
\hline 0.2999 & 0.7001 & 28.2902 & 28.9903 & 9.4301 & 9.6634 \\
\hline 0.3380 & 0.6620 & 17.0877 & 17.7497 & 5.6959 & 5.9166 \\
\hline 0.3626 & 0.6374 & 12.7652 & 13.4026 & 4.2551 & 4.4675 \\
\hline 0.3797 & 0.6203 & 9.7163 & 10.3366 & 3.2388 & 3.4455 \\
\hline 0.3924 & 0.6076 & 8.1262 & 8.7338 & 2.7087 & 2.9113 \\
\hline
\end{tabular}

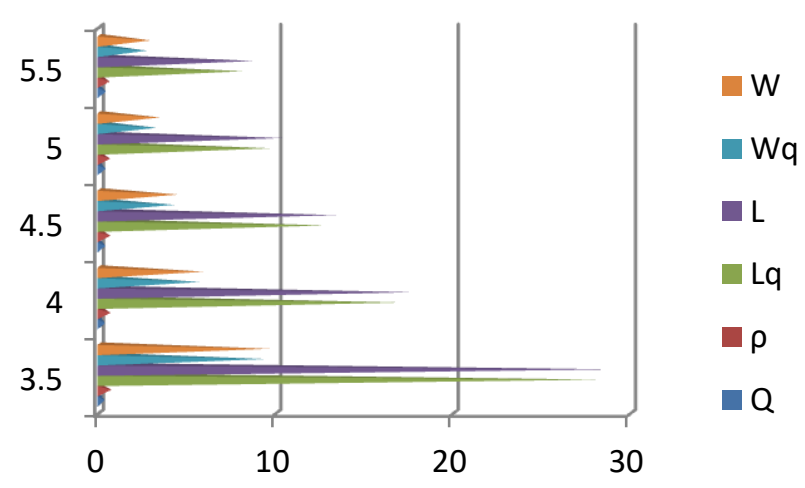

Fig 2: Effect of change of $\gamma$ 


\section{NUMERICAL ANALYSIS}

From the table 1 , it is clear that as the arrival of break down increases, idle time of the server gets lessen and it leads to a service spent by the server gets amplified. Table 2 indicates that, as the probability of completion of repair process gets increased, work gets completed sooner. Hence its corresponding execution measures get decreased.

\section{CONCLUSION}

The above examination gives a reality about the lining issue comprising of different parameters like Balking, reneging, fix process, postpone time and phases of administration occurring in speculation throwing process. The examination completed in the above Queuing issue is to acquire the likelihood creating capacity of the line size and its relating lining execution measures. Numerical examination and pictorial portrayal gives an unmistakable image of the model characterized.

\section{REFERENCES}

1. George Christopher Vosnikas, Stefanos Michael, and Anastasia Vasileiou, "Digital Manufacturing Process chain for one off Replacement Parts", Journal of Manufacturing and Material Processing, pp. 1-17, 2017.

2. S.John, Tu.R.Kelly Foran, Albert.M.Hines, and Paul.R.Aimone, “An Integrated Procedure for Modelling Investment Castings", Journal of Modelling, vol. 47, No.10, pp. 64-68, 1995.

3. Ju Yeon Leo, Joo Seong Yoon, and Bo-Hyun. Kim, “A Big Data Analytics Platform for Smart Factories in Small and Medium Sized Manufacturing Enterprises: An Empirical Case Study of a Die Casting Factory", International Journal of Precision Engineering and Manufacturing, vol. 18, No.18, pp. 1353-1361, 2017.

4. R.F. Khalaf, K. C. Madan \& C.A. Lucas, “An M[x]/G/1 Queue with Bernoulli schedule, General vacation times, random breakdowns, general and general repair times", Applied mathematical sciences,

Vol.5, No.1, 2011, pp.35-51.

5. S. Maragathasundari and K.S. Dhanalakshmi, "Mobile adhoc networks problem-A queueing approach", International journal of communication networks and distributed systems, vol.21, 2018, No.4

6. K.C. Madan and Abu-Dayyeh. A.Z, "On a single server queue with optional phase type server vacations based on exhaustive deterministic service and a single vacation policy", Applied Mathematics and Computation, Vol.149, No.3, 2004, pp. 723-734.

7. K.C. Madan and G. Chodhury, “An $\mathrm{M}^{[\mathrm{x}]} / \mathrm{G} / 1$ queue with Bernoulli vacation schedule under restricted admissibility policy", Sankhaya,Vol.66, 2004, pp.172-193.

8. Omkar Bemblage, and D.Benny Karunakar, "A Study on the Blended Wax Patterns in Investment Casting Process", Proceedings of the World Congress on Engineering, vol. 1, pp. 978-988, 2011.

9. Parlad Kumar, Rupinder Singh, and I.P.S. Ahuja, “A Frame work for Developing a Hybrid Investment Casting Process”, Asian Review of Mechanical Engineering, vol. 2, No.2, pp. 64-68, 2013.

10. W.J. Richards, J.R. Barett, M.E. Springgate and K.C. Shields, "Neutron Radiography Inspection of Investment casting", Appl.Radiat lsot, Vol.61, No.4, pp.75-682, 2004.

11. R.Singh, S.Singh and M.s.J.Hashmi, "Investment Casting", International Journal of Materials in Engineering, Vol.1, No.6, pp.328-334, Dec 1979.

12. S.Jones, and C.Yuan, "Advances in Shell modelling for Investment Casting", Journal of Material Processing, Vol.135, No.2-3, pp.258-265, Apr 2003.

13. S.Wang, A.G.Miranda and C.Shih, "A study of Investment Casting with Plastic Patterns", Journal of Materials and Manufacturing Process, Vol.25, No.12, pp.1482-1488, Dec 2010.

\section{AUTHORS PROFILE}

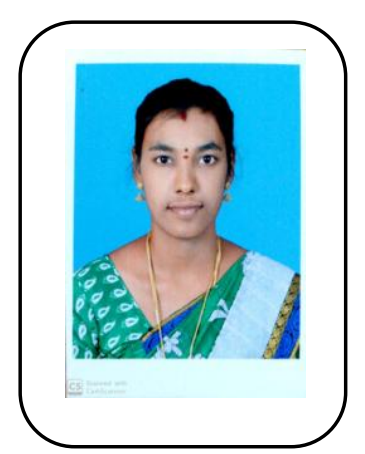

Mrs. R.Vanalakshmi pursued his B.Sc. degree from A.K.D. Dharma Raja Women's College, Rajapalayam in 2009. She got his M.Sc degree from S.F.R. Women's College, Sivakasi in 2011. She obtained his M.Phil. degree from KARE, Krishnankoil in 2018. She has 3 years of teaching experience. Now she is doing research area in Queuing Theory in Kalasalingam Academy of Research and Education, Anand Nagar, Krishnankoil-626126, Tamilnadu, India.

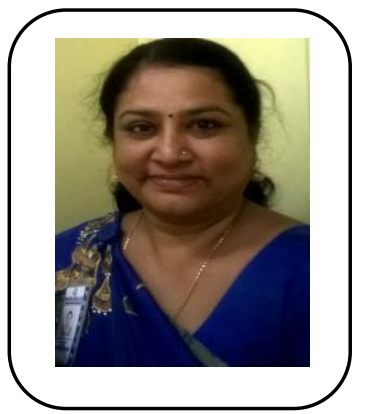

Dr. S. Maragatha Sundari acquired her B.Ed. degree from V.O.C Teachers College, Tuticorin in 1993. She got her M.Sc. in Mathematics from Manonmaniam Sundaranar University; Tirunelveli in 1995 and her M.Phil. degree from Madurai Kamaraj University, Madurai in 2003. She did her Ph.D. in Sathyabama University, Chennai, India. She has more than 17 years of instructing knowledge. She has distributed more than 50 inquire about papers in national and international journals. She has displayed and distributed papers at national and international conferences. She is right now filling in as an Associate Professor in the Department of Mathematics in Kalasalingam Academy of Research And Education, Anand Nagar, Tamilnadu, Krishnankovil-626126, India. In addition, she is doing her research guidance for five research scholars.

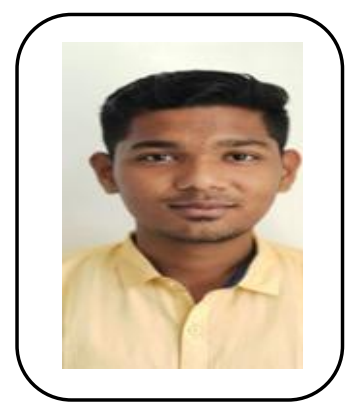

S. Kishore Eswar pursuing his B.Tech Mechanical Engineering in KARE, Krishnankovil. He is a topper of his department and acts as a joint secretary of his department. He has participated in many science competitions and banged many prizes. Now, he is doing research applications in the area of Queuing theory. $\mathrm{He}$ is undergoing industrial implant training in TVS brake linings, Madurai. 